This item was submitted to Loughborough's Research Repository by the author.

Items in Figshare are protected by copyright, with all rights reserved, unless otherwise indicated.

\title{
Visual search behaviour during laparoscopic cadaveric procedures
}

PLEASE CITE THE PUBLISHED VERSION

http://dx.doi.org/10.1117/12.2044389

PUBLISHER

(c) SPIE

VERSION

VoR (Version of Record)

LICENCE

CC BY-NC-ND 4.0

REPOSITORY RECORD

Dong, Leng, Yan Chen, Alastair G. Gale, Benjamin Rees, and Charles Maxwell-Armstrong. 2019. "Visual Search Behaviour During Laparoscopic Cadaveric Procedures". figshare. https://hdl.handle.net/2134/19571. 


\title{
Visual search behaviour during laparoscopic cadaveric procedures.
}

\author{
Leng Dong*a, Yan Chen ${ }^{\mathrm{a}}$, Alastair G. Gale ${ }^{\mathrm{a}}$, Benjamin Rees ${ }^{\mathrm{b}}$, \& Charles Maxwell-Armstrong ${ }^{\mathrm{b}}$ \\ ${ }^{a}$ Applied Vision Research Centre, Loughborough University, Loughborough, UK; \\ ${ }^{\mathrm{b}}$ Queen's Medical Centre, Nottingham, UK
}

\begin{abstract}
Laparoscopic surgery provides a very complex example of medical image interpretation. The task entails: visually examining a display that portrays the laparoscopic procedure from a varying viewpoint; eye-hand co-ordination; complex 3D interpretation of the 2D display imagery; efficient and safe usage of appropriate surgical tools, as well as other factors. Training in laparoscopic surgery typically entails practice using surgical simulators. Another approach is to use cadavers. Viewing previously recorded laparoscopic operations is also a viable additional approach and to examine this a study was undertaken to determine what differences exist between where surgeons look during actual operations and where they look when simply viewing the same pre-recorded operations. It was hypothesised that there would be differences related to the different experimental conditions; however the relative nature of such differences was unknown. The visual search behaviour of two experienced surgeons was recorded as they performed three types of laparoscopic operations on a cadaver. The operations were also digitally recorded. Subsequently they viewed the recording of their operations, again whilst their eye movements were monitored. Differences were found in various eye movement parameters when the two surgeons performed the operations and where they looked when they simply watched the recordings of the operations. It is argued that this reflects the different perceptual motor skills pertinent to the different situations. The relevance of this for surgical training is explored.
\end{abstract}

Keywords: Minimal Access Surgery, eye movement, surgical performance

\section{INTRODUCTION}

Minimal Access Surgery (MAS) is of particular interest to the medical imaging domain as (1) it is in increasing use, (2) comprises viewing a 2D or 3D display and (3) involves complex visual and ergonomic factors. Thus, it poses research challenges in teasing out aspects of MAS surgical expertise and in understanding underlying skills so as to aid in developing training regimes. The need to objectively assess surgical skills has been well documented ${ }^{1,2,3}$. Some of the research investigating such skill development has entailed eye tracking. Unlike radiological imaging, eye tracking in surgery is still very much in its infancy for many reasons, not least including the complexity and potential length of time of each specific operation, issues concerning the actual implementation of eye tracking safely, and ethical issues in recording live operations. Consequently, most such studies have been into the use of surgical simulators ${ }^{4,5,6}$. In general such eye tracking studies span only the recent 10 years or so and do not yet present any complete insight into surgical performance that usefully can be implemented widely in daily clinical practice. We are unaware of any previous eye tracking studies in surgery using cadavers.

A series of comprehensive human factors investigations into surgical performance is currently underway. This paper presents one facet of this programme. MAS operations can easily be recorded and so can be viewed afterwards by many trainees thereby representing a potential valuable training resource. But, is this useful? Elsewhere in these proceedings we describe a study where individuals with different medical skill levels view such recordings whilst their visual behavior was recorded. However, how and where in a display should an experienced surgeon be expected to look? Here we investigated what differences, if any, exist between where and how a surgeon visually searches the displayed laparoscopic anatomy whilst they are performing an operation and where they look if they later simply observe the recording of that same operation, as they would do in a potential training scenario. It was hypothesised that differences would be found, due to the different perceptual-cognitive situations; for instance during an operation where a surgeon looks is directly linked to their on-going manipulation of surgical instruments.

\section{*1.dong@1boro.ac.uk}

Medical Imaging 2014: Image Perception, Observer Performance, and Technology Assessment, edited by Claudia R. Mello-Thoms, Matthew A. Kupinski, Proc. of SPIE Vol. 9037, 903719

(C) 2014 SPIE $\cdot$ CCC code: $1605-7422 / 14 / \$ 18 \cdot$ doi: 10.1117/12.2044389 


\section{METHODS}

The operations investigated took place on a fresh human cadaver during a cadaveric training course ${ }^{7}$. Three laparoscopic procedures, i.e. an appendectomy, cholecystectomy and colectomy, were performed by two surgeons on a human cadaver. During the operations the surgeons' eye movements were recorded. This could have been achieved by several means, such as a head mounted eye tracker or a remote eye movement system. Through discussion with the surgeons before the investigation, it was elicited that the operating surgeon usually makes fairly rapid body movements within a very small area, instead of some larger but more permanent repositioning. Consequently, a Tobii X50 remote eye tracking system was chosen as it allows a small range of head movements whilst not being attached to the surgeon in any way. Also it poses minimum intrusion to the surgeon and the rest of the operating team.

The eye tracker was positioned below the laparoscopic monitor and above and to the side of the cadaver (figure 1) where it could easily record the visual behaviour of the surgeon. At the same time the display output from the laparoscopic camera was recorded. The eye tracking system recorded the visual behaviour of the surgeon with respect to the laparoscopic display. Additionally a scene camera was located so as to record the laparoscopic main monitor and the surgeon's movements. In order to link the surgeon's eye movements to the laparoscopic video then the time stamps of the laparoscopic video and the Tobii video output were matched using information from the scene camera. This then resulted in a video record of each operation which could have overlaid on it the eye movement data of the appropriate operating surgeon. The video records of each operation were then edited down by one of the surgeons so as to encompass the key surgical steps of each operation. This resulted in three videos, 11' 58", 14"'30" and 12'03" in length respectively. These edited videos contained key information such as when an instrument was inserted, operated, or removed, as well as when editing cuts in the video were made.

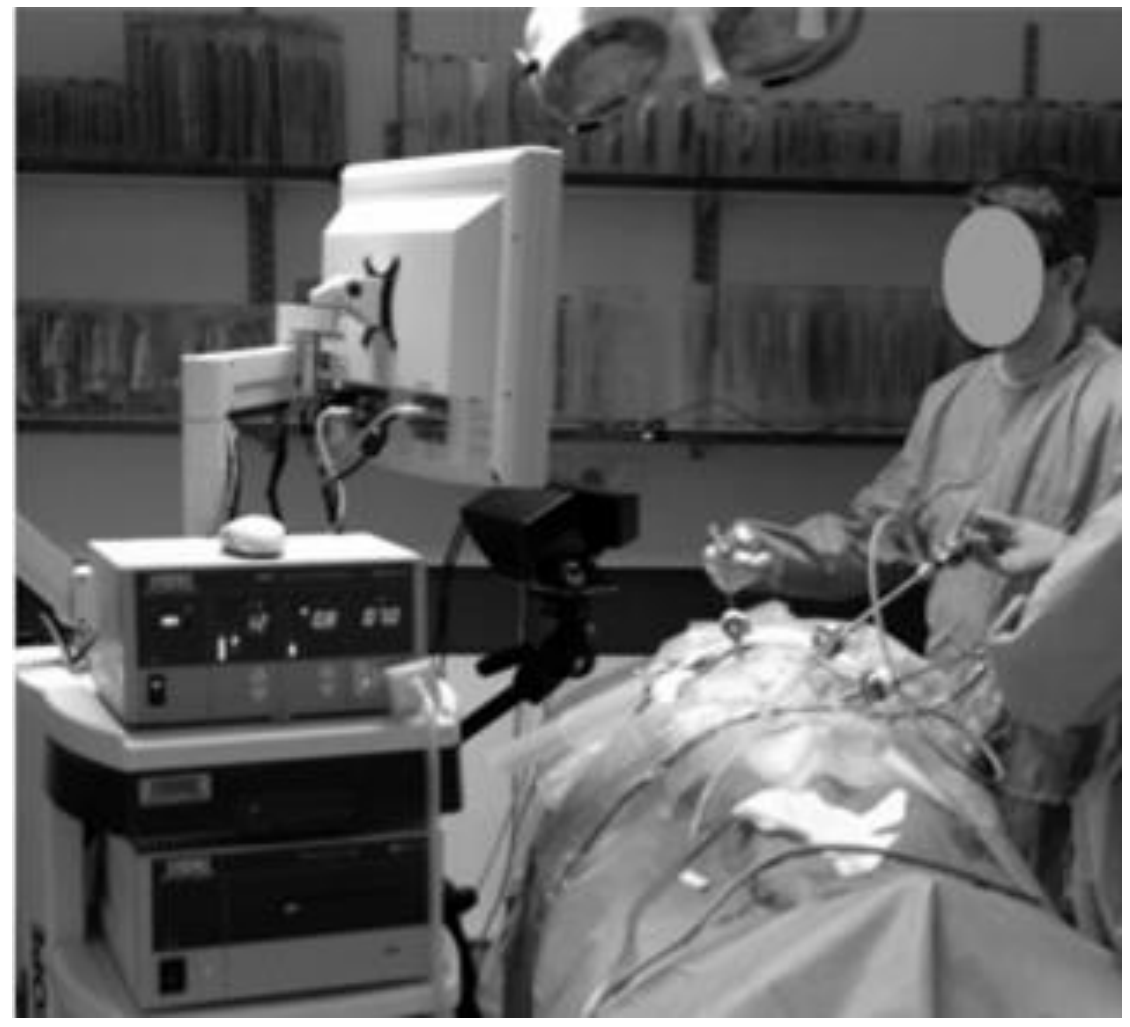

Figure 1 One of the operations in progress with the operating surgeon watching the procedure on the main monitor which has the eye tracker located beneath it. 
Subsequently, six weeks later, the same two surgeons viewed these recordings of their operations without the overlay of their original eye movement data. These videos were presented on a 22-inch display (1,680 x 1,050 resolution) while their visual search behaviour was recorded - again using the Tobii X50 device (figure 2).

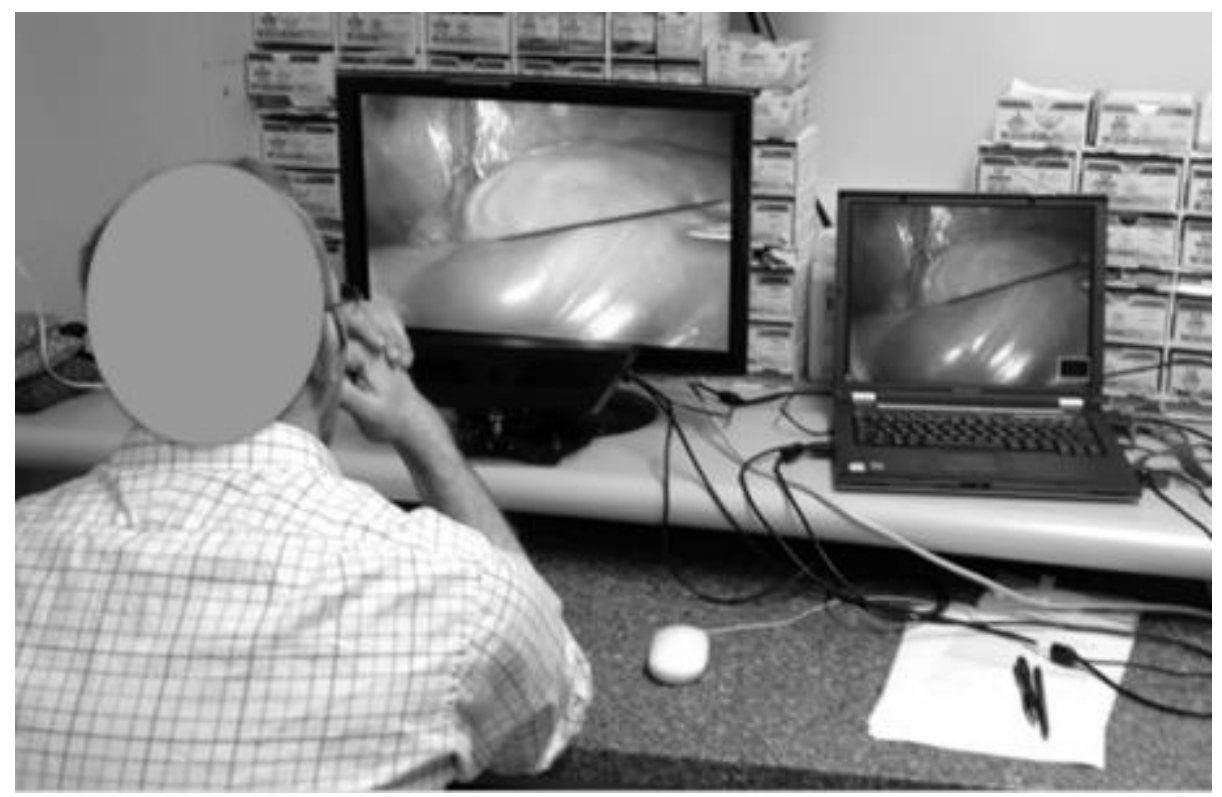

Figure 2. A surgeon viewing a recording of his laparoscopic procedure whilst his eye movements are recorded as shown on the right hand monitor.

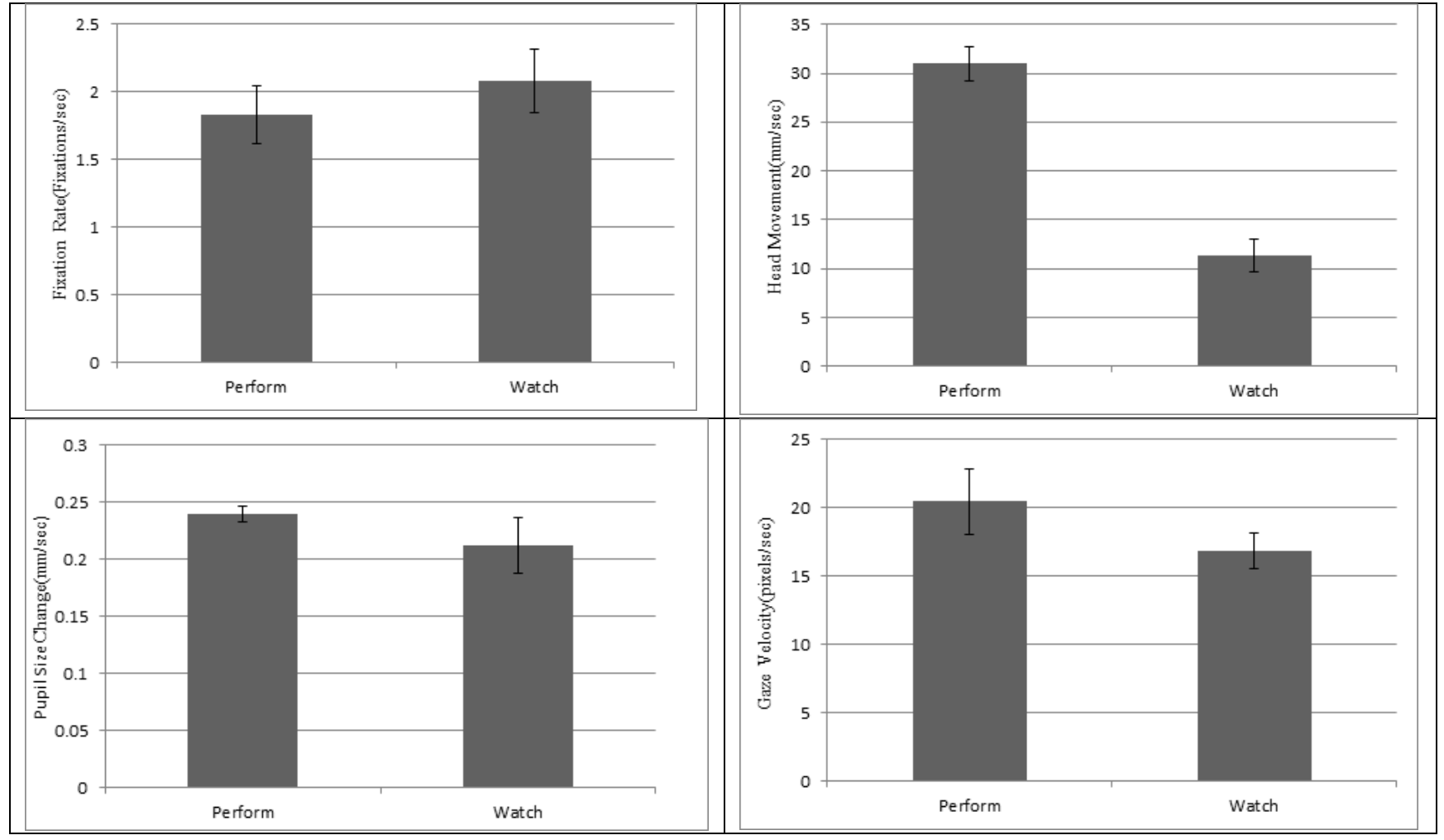

Figure 3. Mean eye parameters comparison between performing the operations and viewing their recordings 


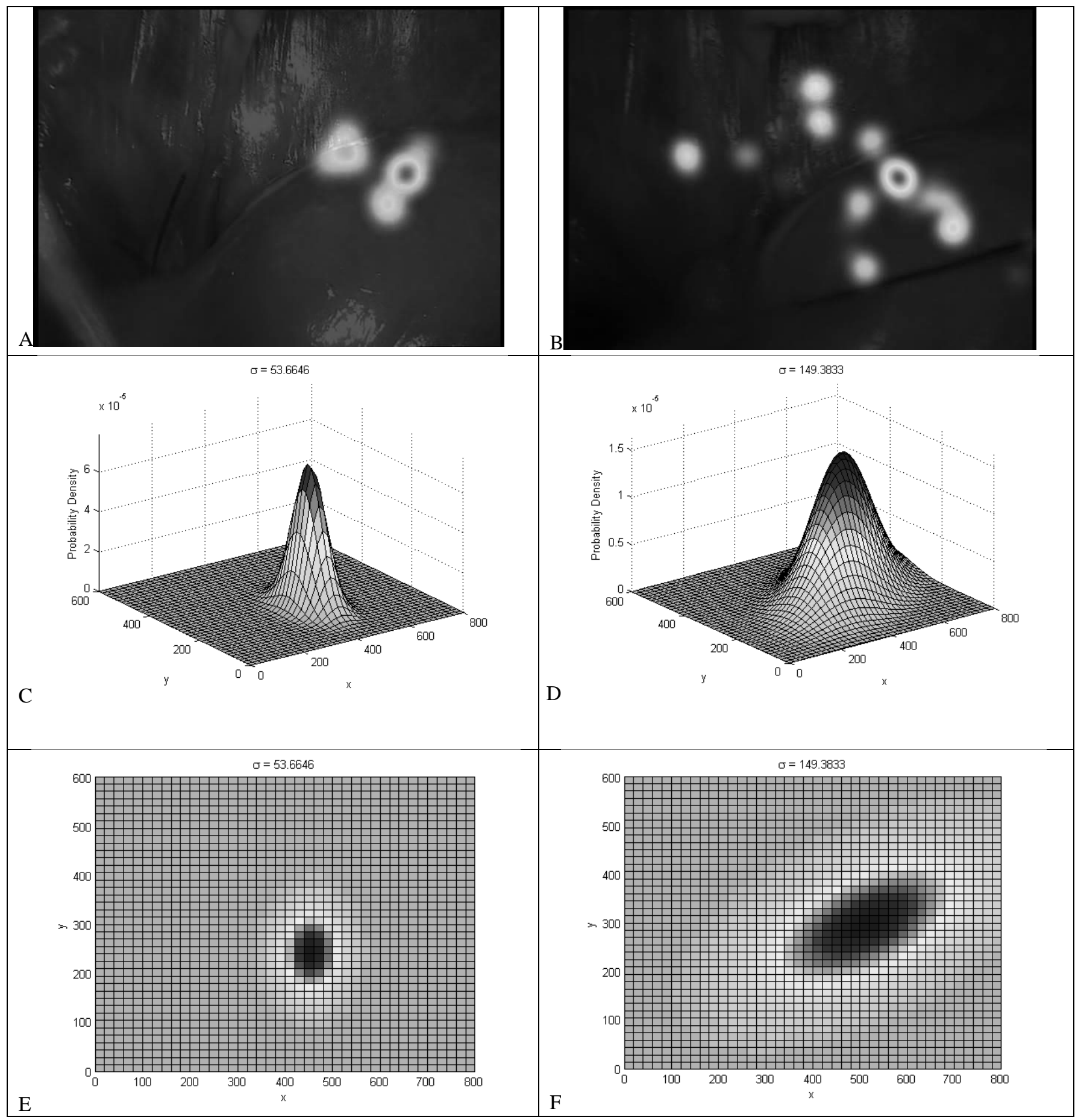

Figure 4. Example of one surgeon's gaze location distribution after an intervention while performing the operation (A) and the surgeon's gaze location distribution while watching the operation recording (B) after the same intervention; normalised 3D distributions of these data while performing the operation $(\mathrm{C})$ and while watching the operation recording (D); respective 2D heat maps of the operating distribution (E) and 2D heat map of such distribution (F).

The resultant eye movement data were then analysed in order to identify any similarities or differences between when the surgeons were simply watching their surgical video and when they were actually performing the surgical procedure. Data were initially examined to assess any overall similarities and differences between the two conditions. The data were also 
analysed in more detail five seconds before and five seconds after a discrete instrument handling action (here termed an intervention) took place in the recorded image stream. There were 12 such interventions across the procedures.

\section{RESULTS}

To take an overall look at the data, gross eye parameters, namely; fixation rate, head velocity, change of pupil size and gaze velocity, were first compared between the two conditions (figure 3). Mean data are shown from the combined overall operations and the combined three edited videos. Somewhat surprisingly the fixation rate is low and similar between the two situations. As would be expected more head movements occur whilst operating, coupled with slightly more pupil size changes. Gaze velocity was higher whilst operating as well. A paired sample t-test was performed on the overall video analysis data of the three tasks. This showed that there was a significant difference in head velocity $(\mathrm{t}(2)=6.288, p<.05, r=.98)$ between the two tasks. Analyses of the other three parameters showed no significance between the operating and viewing data ( $p=$ n.s.).

Intervention analysis

Figure 4 shows an example of the gaze location distribution heat map (5 seconds) by one surgeon after the same intervention when he was performing the operation and when he was watching the recording. Clearly during the operation the surgeon concentrated more on a specific area than when subsequently just viewing the operation video.

The average distance to the centre of the gaze distribution during the 5 seconds in this example is $\sigma=53.66$ for performing the operation example and $\sigma=149.38$ for watching the recording. For each participant, both the mean value of the centre of the gaze distribution and its dispersal before and after each intervention were calculated and compared (Figure 5). A paired sample t-test was performed but no significant results were found $(\mathrm{t}(2)=-2.54, p=\mathrm{n}$.s., $r=.87$ ). Similar analyses were made, looking at one second before and after an intervention but again no significant differences were found.

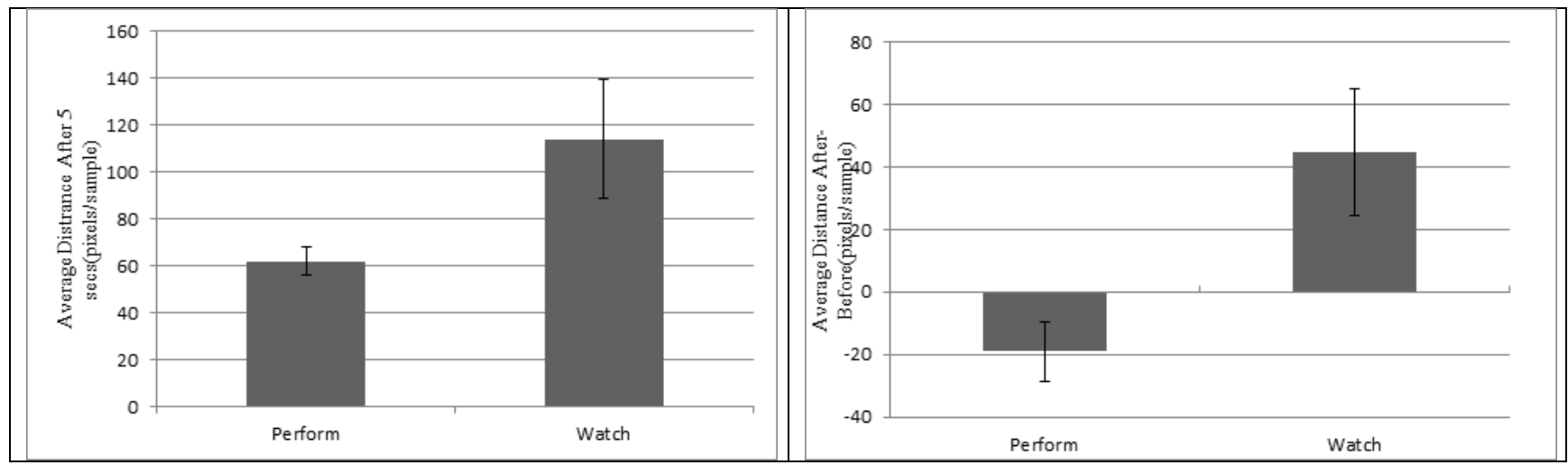

Figure 5 mean distributions of gaze before and after interventions. Figure 6. See text for explanation

The change in eye gaze behavior in the five seconds before and after an intervention was examined and figure 6 shows the differences in these values when operating and just viewing the videos. A paired sample t-test was performed but no significant results were found $(\mathrm{t}(2)=-2.14, p=$ n.s., $r=.83)$. When operating, the surgeons moved their eyes less after an intervention as compared to afterwards. In contrast when simply watching the videos they moved their eyes more afterwards.

Differences in eye movement parameters were found between when the two surgeons performed the operations and when they simply watched the recordings of the operations. This has implications for surgery training. More detailed analyses of these recorded data concerning when changes occur in the video stream as well as when a change in surgical steps occurs are currently still on-going. It is argued that the information from this study provides further insight into analysing performance and visual search behaviour in very dynamic medical imaging scenarios. 


\section{DISCUSSION}

This was a first attempt to examine potential differences between surgeons' eye movements whilst operating and when just viewing the recorded operation imagery. Here there is clearly little overall difference in the measures examined to date between the two situations. Considering the interventions - when just viewing the video imagery there was more widespread visual coverage of the display than when operating. This is as would be expected. In numerous real world tasks a shift of gaze has been found to precede an associated action usually by less than a second ${ }^{8}$. Here the analyses to date suggest support for this from the distribution of gaze behavior data. More detailed analyses will examine this further.

Overall these initial data show the similarities and differences in visual behaviour between the two situations. It is suggested that the eye movement data from surgeons watching pre-recorded operations could be utilised as a potential measure of the development of skilled behaviour. Thus trainees could benefit both from operating on cadavers as well as by watching recordings of such prior operations.

\section{CONCLUSION}

A potential form of laparoscopic surgical training is simply to watch recordings of previous surgical procedures. It is difficult to assess whether this is indeed useful or how this affects the surgeon's developing surgical skill level. This eye tracking study shows how similar a surgeon's visual examination of the real laparoscopic information is to the recorded laparoscopic imagery.

\section{References}

[1] Darzi A., Smith S. \& Taffinder N., Assessing operative skill, BMJ. 1999 April 3; 318(7188): 887-888. .

[2] Moorthy K, Munz Y, Sarker SK, \& Darzi A. Objective assessment of technical skills in surgery, BMJ, 2003 1; 327 (7422):1032-7.

[3] Faurie C, Khadra M. Technical competence in surgeons. ANZ J Surg., 2012 Oct; 82(10):682-90

[4] Tien G., Zheng B., \& Swindells C., Measuring situation awareness of surgeons in laparoscopic training, Proceedings of the 2010 Symposium on Eye-Tracking Research \& Applications, ACM, New York, 2010, 149-152

[5] Law B., Atkins M.S., Kirkpatrick A.E., \& Lomax A.J. Eye gaze patterns differentiate novice and experts in a virtual laparoscopic surgery training environment. Proceedings of the 2004 symposium on Eye tracking research \& applications, ACM, New York, 2004, 41-48.

[6] Wilson M., McGrath J., Vine S., Brewer J., Defriand D. \& Masters R., Psychomotor control in a virtual laparoscopic surgery training environment: gaze parameters differentiate novices from experts. Surg. Endosc., 2010, 24, 2458 2464.

[7] Lloyd, G.M., Maxwell-Armstrong, C., Acheson A.G. Fresh frozen cadavers: an underutilized resource in laparoscopic colorectal training in the United Kingdom, Colorectal Disease, 2011, Vol 13 (9), e303-e304

[8] Land M.F., Vision, eye movements, and natural behaviour Visual Neuroscience (2009), 26, 51-62. 